\title{
Chronic Inflammatory Demyelinating Polyradiculoneuropathy 101-Pitfalls and Pearls of Diagnosis and Treatment
}

\author{
Said R Beydoun, ${ }^{1}$ Thomas H Brannagan III, ${ }^{2}$ Peter Donofrio, ${ }^{3}$ Carol Lee Koski ${ }^{4}$ and Eric Lancaster ${ }^{5}$
}

1. Neuromuscular Division, Keck Medical Center of University of Southern California, US; 2. Department of Neurology, Neurological Institute, Columbia University, New York, New York, US; 3. Vanderbilt University Medical Center, Nashville, Tennessee, US; 4. GBS/CIDP Foundation International, Narberth, Pennsylvania, US; 5. The University of Pennsylvania, Philadelphia, Pennsylvania, US.

$\mathrm{C}$ hronic inflammatory demyelinating polyradiculoneuropathy (CIDP), which is caused by demyelination of the peripheral nerves, is characterized by progressive weakness and impaired sensory function in the arms and legs. CIDP is a treatable condition in which early diagnosis is crucial to limit chronic disability. CIDP can mimic other neuropathies and it is important to identify these in order to ensure prompt treatment. Patients with other causes of neuropathy should be suspected of having CIDP if there is rapid progress or proximal weakness. Intravenous immunoglobulin (IVIG), corticosteroids, and plasma exchange are first-line therapies. The IVIG CIDP Efficacy (ICE) trial, the largest trial reported of any CIDP treatment, demonstrated that IVIG therapy reduced disability and functional impairment, as well as improved quality of life. Autoantibodies against membrane proteins of the peripheral nerve axons or the myelin sheath have been reported recently, and an improved understanding of antibody responses in CIDP may enable the development of future targeted therapeutic interventions.

\section{Keywords}

Chronic inflammatory demyelinating polyradiculoneuropathy (CIDP), differential diagnosis, intravenous immunoglobulin (IVIG), corticosteroids, plasma exchange

\footnotetext{
Disclosure: Said R Beydoun has received research grants from CSL Behring and is a consultant/speaker for Grifols and Baxalta. Thomas H Brannagan II has served as a consultant/speaker for Grifols, a consultant for Shire and has received clinical trial support from Novartis. Peter Donofrio has served on advisory boards for CSL Behring UCB Pharma and Grifols. Carol Lee Koski has served as a speaker for Grifols and CSL and as a Medical Advisor for GBS CIDP FI. She is also a Member of Safety committee for CSL on SubQ trial CIDP. Eric Lancaster has received grant support and course teaching from Grifols, Inc. He has carried out consulting for Amgen, Jaansen, Medimmune, and has been involved in writing expert reports and testifying for the Vaccine Injury Compensation program.

Authorship: All named authors meet the International Committee of Medical Journal Editors (ICMJE) criteria for authorship of this manuscript, take responsibility for the integrity of the work as a whole, and have given final approval to the version to be published.

Open Access: This article is published under the Creative Commons Attribution Noncommercial License, which permits any noncommercial use, distribution, adaptation, and reproduction provided the original author(s) and source are given appropriate credit.

Received: November 10, 2016

Accepted: January 05, 2017

Citation: US Neurology, 2017;13(1):18-25

Corresponding Author: Said R Beydoun, USC Health Care Consultation Center II, 1520 San San Pablo Street, Suite 3000, Los Angeles, California, US 90033. E: sbeydoun@usc.edu
}

Support: The publication of this article was supported by Grifols. The views and opinions expressed in the article are those of the authors and not necessarily those of Grifols. US/GX/0316/0286
Chronic inflammatory demyelinating polyradiculoneuropathy (CIDP) is an autoimmune demyelinating polyradiculoneuropathy characterized by chronically progressive weakness and impaired sensory function in the lower and upper extremities. ${ }^{1}$ Symptoms, which are progressive over at least 8 weeks, may include weakness of the arms and legs (both proximal and distal), loss of vibration and joint position sense, poor balance, numbness, paresthesias, and loss of deep tendon reflexes (areflexia). Cranial nerves (other than cranial nerve V or VII) and autonomic functions are generally preserved. ${ }^{2}$ The phenotype of symmetrical proximal and distal motor and sensory symptoms and signs define typical CIDP. Atypical CIDP includes other clinical presentations, such as asymmetric, multifocal motor and sensory symptoms, distal sensory, or predominantly motor or sensory types. Moreover, up to $16 \%$ of patients with CIDP may demonstrate acute-onset CIDP, which is characterized by a rapidly progressive onset within 8 weeks. ${ }^{3,4}$

The exact mechanisms that underlie the development of CIDP have not been elucidated fully, although evidence suggests likely contributions by both cellular and humoral factors. It is twice as common in men, with increasing frequency after age 60, although it can occur at any age. ${ }^{5}$ The incidence and prevalence of CIDP have been estimated at 1.6/100,000/year to $8.9 / 100,000$, respectively. ${ }^{6}$ There are many phenotypic variants of CIDP (Table 1), which suggests that the disorder may not be a discrete entity, but a spectrum of conditions. ${ }^{7}$ Elevated levels of cerebrospinal fluid (CSF) protein are present in the majority of patients although normal CSF results do not exclude the diagnosis of CIDP. ${ }^{7,8}$ Currently, there are no well-established biomarkers, although autoantibodies to contactin-1 and neurofascin-155 define CIDP subsets of patients with specific clinical features. ${ }^{9}$

\section{Diagnosis}

Early diagnosis is vital for this treatable condition in order to limit disability as a result of secondary axonal damage. Initial diagnostic criteria, including 
Table 1: Major phenotypical variants of chronic inflammatory demyelinating polyradiculoneuropathy ${ }^{7}$

\begin{tabular}{|l|l|l|l|l|}
\hline CIDP phenotypic variant & $\begin{array}{l}\text { Estimated } \\
\text { prevalence within } \\
\text { CIDP }\end{array}$ & Onset & Clinical symptom & Distribution \\
\hline Typical CIDP & $51 \%$ & Chronic & Sensory and motor & Symmetrical, proximal and distal \\
\hline Sensory CIDP & $4-35 \%$ & Chronic & Sensory predominant, motor involvement may develop & As per typical CIDP \\
\hline $\begin{array}{l}\text { Chronic immune sensory } \\
\text { polyradiculopathy }\end{array}$ & $5-12 \%$ & Chronic & Sensory ataxia & As per typical CIDP \\
\hline $\begin{array}{l}\text { Lewis-Sumner } \\
\text { syndrome/MADSAM }\end{array}$ & $6-15 \%$ & Chronic & Sensory and motor & Asymmetrical, often upper limb onset \\
\hline Focal CIDP & $1 \%$ & Chronic & Sensory and motor & Focal; may progress to diffuse CIDP over time \\
\hline DADS & $2-17 \%$ & Chronic & Sensory predominant, but may include motor involvement & Symmetrical, distal \\
\hline Acute onset CIDP & $2-16 \%$ & Acute onset & As per typical CIDP & As per typical CIDP \\
\hline Motor CIDP & $4-10 \%$ & Chronic & Motor predominant & As per typical CIDP \\
\hline
\end{tabular}

CIDP = chronic inflammatory demyelinating polyradiculoneuropathy; DADS = distal acquired demyelinating symmetric; MADSAM = multifocal acquired demyelinating sensory and motor neuropathy.

the American Academy of Neurology and Inflammatory Neuropathy Cause and Treatment (INCAT) criteria, were designed for research and have a high specificity, but low sensitivity for CIDP. For this reason, many patients do not meet the diagnostic criteria and do not receive the appropriate treatment. ${ }^{10,11}$ More recently, diagnostic criteria for use in clinical practice have been developed, including the European Federation of Neurological Sciences (EFNS)/Peripheral Nerve Society (PNS), Neuropathy Association, and the Koski criteria. According to the EFNS/PNS criteria, CIDP should be considered in a patient if there is clinical evidence for a progressive symmetrical or asymmetrical polyradiculoneuropathy and a clinical course that is relapsing and remitting or progresses for $>2$ months. ${ }^{12}$ Electrodiagnostic testing is essential to make the diagnosis, EFNS/PNS electrodiagnostic criteria for definite or probable diagnosis of CIDP require the presence of demyelinating findings (DF) in at least 2 nerves; for possible CIDP, abnormality may need to be evident only in 1 nerve. ${ }^{10}$ The DF can include any of those abnormal parameters: Prolongation of distal motor latency $(>50 \%)$, slowing of conduction velocity $(<30 \%)$, absence or prolongation of $\mathrm{F}$ response latencies, presence of partial conduction block (50\% for definite, 30\% for probable), and abnormal temporal dispersion ( $>30 \%$ prolongation of CMAP duration between distal and proximal CMAP). Preliminary evidence suggests that more extensive testing such as 8 motor nerves ${ }^{13}$ or 3- rather than 2-limb testing may increase the diagnostic sensitivity for definite CIDP, particularly in individuals with atypical (asymmetric and distal) phenotypes, which comprised $75.5 \%$ (40 of 53) of the study cohort. ${ }^{14}$

With unilateral, forearm/foreleg, four-nerve studies the EFNS/PNS criteria has been reported to provide a sensitivity of $81.3 \%$ and specificity of $96.2 \%$ for "definite/probable" CIDP. ${ }^{15}$ Supportive criteria include an elevated CSF protein with leukocyte count $<10 / \mathrm{mm}^{3}$ per high powered field, magnetic resonance imaging (MRI) of the nerve roots, nerve biopsy, and treatment response to immunomodulatory therapy. For diagnosis, objective measures should be used to verify and apply the criterion of treatment response. Electrodiagnostic studies including sensory and motor nerve conduction studies should be performed; studies may need to be performed bilaterally, or use proximal stimulation in motor nerves in order to document multifocal demyelination. In the Koski criteria, according to a classification rule, which was derived by a classification and regression tree analysis and applied to
150 patients, the diagnosis of CIDP required that a patient had a chronic nongenetic polyneuropathy, progressive for at least 8 weeks, without a serum paraprotein and either: ${ }^{16}$

- recordable compound muscle action potentials in $\geq 75 \%$ of motor nerves tested and either an abnormal distal latency in $>50 \%$ of nerves or abnormal motor conduction velocity in $>50 \%$ of nerves or abnormal F wave latency in $>50 \%$ of nerves;

- symmetrical onset of weakness, symmetrical weakness in all four limbs and proximal weakness in $\geq 0 n e$ limb.

The Koski criteria have $50-83 \%$ sensitivity and 89-97\% specificity for typical presentations of CIDP. ${ }^{15-17}$

\section{Chronic inflammatory demyelinating polyradiculoneuropathy and diabetes mellitus}

An association between CIDP and diabetes mellitus (DM) has been reported. Type $2 \mathrm{DM}$ (T2DM) is typically increased in the older population in which CIDP occurs most frequently. However, it is not known whether DM is a major risk for CIDP. Using an epidemiological approach, based on multiple concurrent cases from an Italian population of 4,334,225, the number of expected individuals with CIDP and associated DM was approximated at 13.03 , which corresponded to a standardized morbidity ratio (SMR) of 1.07 (95\% confidence interval [CI], 0.58-1.80). ${ }^{18}$ The presence of DM was assessed using the data reported in the clinical records of each patient (clinical history, fasting blood glucose, or reported use of anti-diabetes drugs). In total, 155 patients with CIDP were identified, 14 of whom were also affected by DM (type 1 or 2). An investigation of incidence and prevalence in Olmsted Country (1581 medical records) identified 23 patients with CIDP (19 definite and 4 probable). The incidence of CIDP was 1.6/100,000/year and the prevalence was $8.9 / 100,000$ persons on January 1,2000 . Only one of the 23 CIDP patients (4\%) also had DM, whereas 14 of 115 age- and sexmatched controls (12\%) had DM. ${ }^{6}$

The findings of these studies therefore do not support an increased incidence of DM in patients with CIDP. ${ }^{6,18}$ However, it is possible that some CIDP patients were not identified or that some CIDP cases with associated DM, or preclinical DM, were missed. CIDP may occur 
with equal frequency in patients with types 1 and $2 \mathrm{DM}^{19}$ presenting a diagnostic challenge: both CIDP and diabetes may result in elevated CSF protein..$^{20}$ Pathologic evaluation clearly differentiates diabetic lumbosacral radiculoplexus neuropathy (DLRPN) from CIDP. These patients would not meet electrophysiologic or clinical criteria for CIDP. This study concluded that the painless motor neuropathy seen in diabetic patients represents painless DLRPN and not CIDP. ${ }^{21}$ However, a consideration of concomitant CIDP in patients with DM is important since treatment of demyelinating neuropathy would limit disability. It is key to consider a diagnosis of CIDP when diabetic patients develop relatively symmetrical proximal weakness or rapid worsening of neuropathy despite good glycemic control.

In an observational, retrospective study of CIDP patients with ( $n=67)$ and without $(n=67) D M$, those with concomitant DM showed more severe clinical and electrophysiological neuropathy, based on higher lower limb vibration potential thresholds $(p=0.004)$, higher Toronto Clinical Neuropathy Scores $(p=0.0009)$, more proximal weakness $(p=0.03)$, more gait abnormalities $(p=0.03)$, and more abnormal nerve conduction study findings. Subjects with CIDP and DM also had more abnormal sural nerve conduction studies with lower sural sensory nerve action potential amplitudes $(2.4 \pm 3.0 \mu \mathrm{V}, 6.6 \pm 6.0 \mu \mathrm{V}, \mathrm{p}<0.0001)$ and slower sural nerve conduction velocities $\left(38.6 \pm 5.4 \mathrm{~m} / \mathrm{s}, 41.0 \pm 5.3 \mathrm{~m} / \mathrm{s}, \mathrm{p}=0.04 .{ }^{22}\right.$ Patients with $\mathrm{DM}$ were less likely to receive specific/disease-modifying therapy although their response rates to CIDP treatment were similar in comparison with those who did not have DM. In particular, the duration of neuropathy rather than the DM status was associated with treatment response. Responders had a shorter CIDP duration than the nonresponders $(8.0 \pm 6.0$ years versus $11.9 \pm 7.6$ years, $p=0.004)$.

\section{Differential Diagnosis}

Neurological examination investigating sensory, motor, and autonomic signs help to define the topography and nature of neuropathy. Principal laboratory studies to support of the diagnosis of CIDP are CSF examination, nerve conduction studies, and nerve biopsy. ${ }^{23}$ If a paraprotein is detected on serum immunofixation or serum kappa lambda free light chain ratio, a Iymphoproliferative disorder, such as osteosclerotic myeloma or lymphoma, should be considered. ${ }^{23}$

There is no biomarker for CIDP and differential diagnosis can be challenging as hereditary, toxic, metabolic, and neoplastic neuropathies must be considered. In a retrospective study of 59 patients who had been referred with a diagnosis of CIDP, patients were classified into whether or not they had CIDP according to the EFNS/PNS criteria. ${ }^{24}$ Nearly half (47\%) of these patients failed to meet the minimal EFNS/ PNS diagnostic requirements. Another study of patients, diagnosed with CIDP and referred after not responding to initial trials of treatment, found that $54 \%$ of patients had an alternative diagnosis, the most common of which was amyotrophic lateral sclerosis. Forty-six percent of refractory patients had CIDP though, and the majority responded to escalating immunotherapy. ${ }^{25}$ Common diagnostic errors included dependence on subjective treatment benefit, imprecise electrophysiological interpretation of demyelination, and placing too much importance on mild or moderate cytoalbuminologic dissociation. There is also a large group of patients with presumed CIDP, who do not fulfill any of the proposed electrodiagnostic criteria. ${ }^{26}$ Examples of some confounding diagnoses include:
Guillain-Barré syndrome (GBS) is a monophasic disorder that includes a spectrum of acute autoimmune peripheral neuropathies. It is less common than CIDP and frequently preceded by a triggering event including viral and diarrheal illness. CIDP has a much slower onset than GBS and a more chronic course. The first symptoms in GBS are typically pain, numbness, paresthesia, or weakness in the limbs. ${ }^{27,28}$ Main features include rapidly progressive bilateral and relatively symmetric weakness of the limbs and reduced or absent tendon reflexes in the affected extremities. Respiratory muscles, cranial nerves, and autonomic nerves are often affected in GBS. ${ }^{28}$ Patients with CIDP may show a GBS-like onset and a CIDP diagnosis should be considered when a patient thought to have GBS relapses or progresses beyond 8 weeks from onset or when deterioration occurs three times or more.

Multifocal motor neuropathy ( $M M N$ ) is an acquired immune-mediated neuropathy that is characterized by chronic or stepwise progressive asymmetrical weakness without sensory deficits. In contrast to CIDP, the motor deficit in MMN tends to be predominantly in the arms, distal more than proximally, and an asymmetric or multifocal nerve involvement. MMN is characterized electrophysiologically by multifocal conduction block across non-entrapment sites with preservation of sensory conduction in the affected nerve segment. At least half the patients have a polyclonal-immunoglobulin (Ig) M anti-ganglioside antibody, and CSF protein is usually normal or slightly elevated. Abnormal MRI T2 signal of the brachial plexus has been reported to occur in $30-40 \%$ of patients with MMN who have distal upper extremity weakness. ${ }^{2}$ Neuropathy ultrasound may be a useful addition as a diagnostic tool. ${ }^{29}$

Distal, Acquired Demyelinating Symmetric (DADS) neuropathy with myelin-associated-glycoprotein (MAG) antibody: CIDP is typically characterized by proximal and distal weakness, but CIDP variants include a distal phenotype. The variant of DADS with no MAG antibody is a form of atypical CIDP. Differential diagnosis of DADS neuropathy includes neuropathy associated with an IgM monoclonal protein binding to MAG antibody, though some have paraprotein without MAG reactivity. IgM MAG neuropathy is not considered to be an atypical CIDP but another entity, which does not respond to first-line treatment for CIDP. ${ }^{30}$

Chronic ataxic neuropathy with ophthalmoplegia, M-protein, cold agglutinins and disialosyl antibodies (CANOMAD) is a rare disorder with severe sensory ataxia and cranial nerve involvement. ${ }^{31}$ This includes ophthalmoplegia, dysphagia, or dysarthria and minimal weakness CANOMAD typically progresses over years and is associated with IgM antibodies to ganglioside disialosyl moieties, such as GD1b.

Polyneuropathy, Organomegaly, Endocrinology, Monoclonal gammopathy and Skin change (POEMS): in a review of data collected between 2000 and 2010, 60\% of patients with POEMS syndrome had originally been diagnosed with CIDP because, in many patients with POEMS syndrome, the other manifestations are subtle, and the primary problem is the demyelinating neuropathy. ${ }^{32}$ Correct diagnosis was only made after the patients failed to respond to the standard treatment. POEMS syndrome is associated with plasma cell dyscrasia of an IgA or IgG lambda paraprotein and a spectrum of multisystem clinical features. ${ }^{33}$ It presents with neuropathy typified by motor and sensory 
involvement with axonal and demyelinating features. Neuropathic pain may be present ${ }^{32}$ and pain in the legs is found in $76 \%$ of patients with POEMS syndrome compared with only $7 \%$ of patients with CIDP. ${ }^{32}$ POEMS syndrome is typically associated with osteosclerotic myeloma, and, occasionally, with Castleman disease or monoclonal gammopathy of unknown significance. ${ }^{34,35}$ POEMS syndrome can sometimes be distinguished from CIDP by the clinical profile, ${ }^{32}$ as well as the presence of a lambda monoclonal protein. On electrodiagnosis, demyelinating changes occur typically in CIDP in a multifocal pattern with more uniform changes in POEMS syndrome. ${ }^{36}$ Elevated levels of vascular endothelial growth factor may also be helpful in making a diagnosis of POEMS syndrome. A skeletal survey is recommended in patients with a demyelinating polyneuropathy and a lambda monoclonal protein to detect osteosclerotic lesions. ${ }^{37-39}$

\section{Treatment \\ Corticosteroids}

The EFNS/PNS guidelines recommend that a trial of corticosteroids, IVIG, or plasmapheresis should be considered in all patients with significant disability. ${ }^{12}$ Supporting evidence in an unblinded, randomized, controlled trial $(n=28)$, showed that prednisone treatment led to a small but significant improvement over no treatment in scored neurological disability, some measures of computer-assisted sensory detection threshold, graded muscle strength, and some attributes of nerve conduction. ${ }^{00,41}$ Pulsed oral dexamethasone therapy showed equal efficacy to oral prednisolone in a 6-month randomized clinical trial. ${ }^{42}$ Steroids are accepted as firstline therapy for those patients with sensory and motor dysfunction who can tolerate steroids but should not be used in patients who present with motor variant CIDP, in which corticosteroids are reported to cause worsening. ${ }^{12,43,44}$ Pure motor CIDP can sometimes be confused with MMN, in which corticosteroid treatment can also result in clinical worsening. The long-term use of corticosteroids is associated with numerous side effects, some serious. These include osteoporosis, skin fragility, weight gain, diabetes, worsening hypertension, hip fractures, cataracts, sleeplessness, and cushingoid appearance. ${ }^{41}$

\section{Intravenous immunoglobulin}

IVIG has been an accepted as first-line therapy for the treatment of CIDP over the last 20 years and is supported by Class I evidence. ${ }^{12,45}$ The largest trial reported of any CIDP treatment is the ICE trial, which is a randomized, double-blind, placebo-controlled, response-conditional crossover trial of 117 patients with CIDP (Figure 1). ${ }^{46}$ The aim of the ICE trial was to establish whether immune globulin injection (human), 10\%, caprylate/ chromatography purified (IVIG-C; GAMUNEX ${ }^{\circledR}-\mathrm{C}$, Grifols Therapeutics Inc., Clayton, North Carolina, US) has short- and long-term benefit. The trial utilized a baseline loading dose of $2 \mathrm{~g} / \mathrm{kg}$ over $2-4$ days and then a maintenance infusion of $1 \mathrm{~g} / \mathrm{kg}$ over 1-2 days, every 3 weeks, for up to 24 weeks. Participants who completed the first period or crossover period and whose improved INCAT disability score was consistently $\geq 1$ point greater than at baseline were eligible for inclusion in a 24week, double-blind extension phase. Eligible participants were randomly re-assigned in a 1:1 ratio to receive $1 \mathrm{~g} / \mathrm{kg}$ IVIG or placebo over 1-2 days, every 3 weeks, for up to 24 weeks (no loading dose was given), and the adjusted INCAT disability score was assessed every 3 weeks during this period. The primary outcome measure was the INCAT disability score, which is a 10-point ordinal measure that captures changes in daily arm and leg activities and mobility. Secondary outcome measures included grip
Figure 1: ICE study design ${ }^{46}$

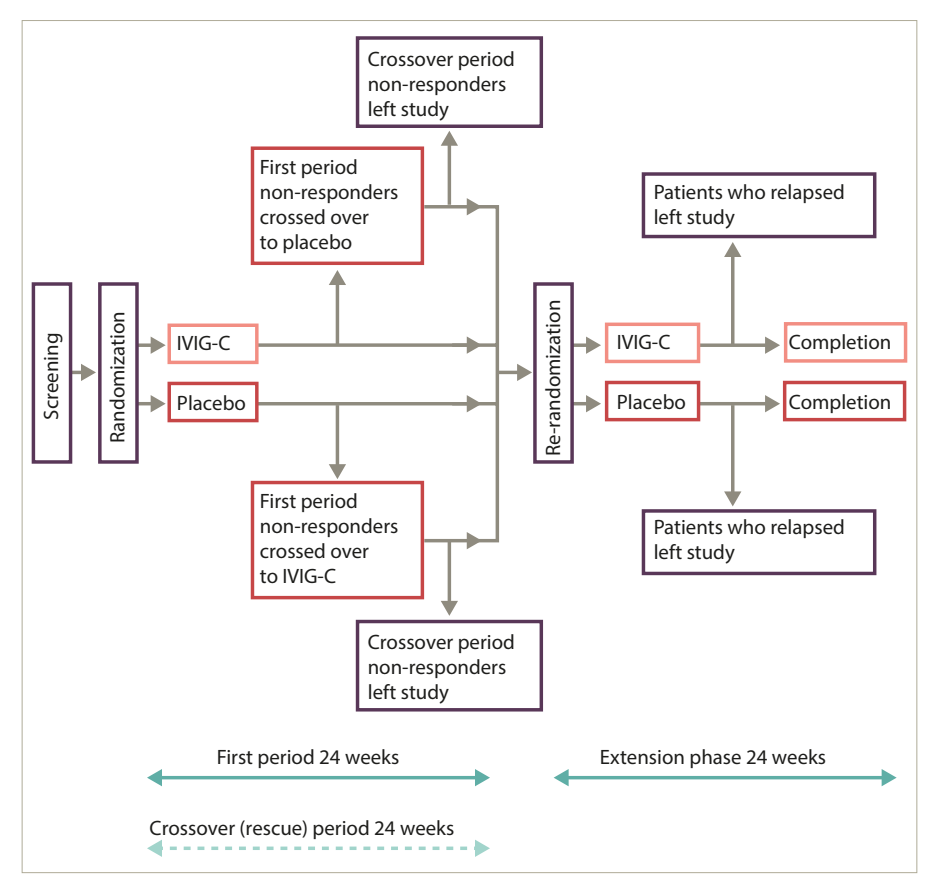

strength; Medical Research Council (MRC) score, an evaluation of muscle strength; and 36-item short-form survey (SF-36), a quality of life measure. ${ }^{47}$ During the initial period 54\% (32 out of 59) patients treated with IVIG showed improvement in adjusted INCAT disability score, a measure of activities of daily living function, that was maintained to week 24 compared with $21 \%$ (12 out of 58) who achieved improvement with placebo (treatment difference: 33.5\%, 95\% Cl 15.4-51.7; $p=0.0002$ ). Similar findings were observed during the cross-over period. During the extension phase (6-month follow-up) those who continued to receive IVIG showed decreased relapse compared with those who had received placebo ( $p=0.011)$. A significant improvement was seen earlier in grip strength (as early as day 16) compared with changes on the INCAT disability scale in patients who had received IVIG versus those who were given placebo. ${ }^{48}$

\section{Timing of response to intravenous immunoglobulin}

In the ICE trial, 58 patients received placebo and 59 received IVIG administered as a $2 \mathrm{~g} / \mathrm{kg}$ loading dose over 2-4 days followed by a maintenance dose of $1 \mathrm{~g} / \mathrm{kg}$ over 1-2 days every 3 weeks, for up to 24 weeks. Among the 30 patients who responded to IVIG, 14 (47\%) had an improved adjusted INCAT score by week 3. A further 16 (53\%) improved after a second infusion at week 6 . Although not considered responders in the ICE trial, an additional two patients improved with IVIG beyond the 6-week window; the latter was an a priori stipulation of response in the ICE trial. In clinical practice, patients may take longer than 6 weeks to respond. The novel response-conditional, crossover study design of the ICE trial required that patients cross over to the alternative treatment if they failed to improve or at the first sign of deterioration or if they were unable to maintain improvement at any time after 6 weeks (Figure 1). ${ }^{49}$ This design addressed concerns about lack of clinical equipoise, which were raised by the physicians interested in participating in the trial. ${ }^{49}$ The magnitude of change in CIDP outcome measures required to correlate with a perception of a clinical improvement in the ICE trial has been described through minimum clinically important differences analysis. ${ }^{50}$ 
Adverse events related to intravenous immunoglobulin In the ICE trial, the most common adverse reactions with GAMUNEX ${ }^{\circledR}-\mathrm{C}$ (immune globulin injection [human], 10\% caprylate/chromatography purified) were headache, fever, chills, hypertension, rash, nausea, and asthenia, and the most serious adverse reaction in clinical studies was pulmonary embolism (PE) in 1 subject with a history of PE. The frequency of adverse events, including serious adverse events, did not seem to depend on age, weight, CIDP severity, or previous IVIG exposure. Although no definitive studies have been carried out exploring mitigation of IVIGrelated side effects, slowing or temporarily discontinuing the infusion and symptomatic therapy with analgesics, nonsteroidal anti-inflammatory drugs, antihistamines, and glucocorticoids may improve some IVIGassociated side effects. ${ }^{51}$

In the ICE study, IVIG also led to health-related quality of life improvements. ${ }^{52}$ In the first period, compared with placebo, greater improvements were observed in both SF-36 physical and mental component score (difference: 4.4 points; $95 \% \mathrm{Cl}$, 0.7-8.0). In addition, participants who received IVIG showed a larger improvement in the Rotterdam Handicap Scale compared with those who received placebo (difference, 3.4 points; 95\% Cl 1.4-5.5; $\mathrm{p}=0.001$ ).

Please see the Important Safety Information about GAMUNEX-C on pages 22-3 and refer to the brief summary of full Prescribing Information ${ }^{53}$ in the Appendix.

\section{Plasma exchange}

Plasma exchange (PLEX) has been established as first-line therapy for CIDP in short-term efficacy studies. PLEX should be considered for initial treatment if patients cannot tolerate corticosteroids or IVIG, or have continued to deteriorate following IVIG or corticosteroids. ${ }^{12}$ The temporal effect is short term, and an indwelling apheresis catheter may be required. Two small double-blind, sham-controlled, randomized clinical trials totaling 47 participants showed that PLEX provides short-term benefit in around two-thirds of patients but that rapid deterioration often ensues when the PLEX is stopped. ${ }^{54-56}$ The use of PLEX is associated with adverse events related to difficulty with venous access, use of citrate, and haemodynamic changes. ${ }^{57}$

\section{Immunosuppressive agents}

The EFNS/PNS guidelines conclude that more research is needed before evidence-based recommendation on immunosuppressive treatment can be made; however, these treatments, including IV pulse cyclophosphamide, ${ }^{57,58}$ may be considered when the response to corticosteroids, IVIG, or PLEX is inadequate. ${ }^{12}$ Although there have been reports on their potential use by case report or open-label studies, randomized, controlled clinical trials to date have not established efficacy of such agents as primary or as add-on agents in the treatment of CIDP. 59,60

\section{Risk of relapse}

Electrodiagnostic studies have been carried out on participants in the ICE trial who responded to treatment in the first period and were subsequently re-randomized to placebo in the 24-week extension phase. These data indicated that an increase in the total number of DF, specifically conduction block, may signal an increased risk of relapse after discontinuation of therapy whereas the absence of new demyelination may suggest a decreased risk of subsequent relapse. ${ }^{0}$
Autoantigens in chronic inflammatory demyelinating polyradiculoneuropathy

Recently, autoantibodies against membrane proteins of the peripheral nerve axons or the myelin sheath have been reported in CIDP. For example, a major component of myelin, protein zero (P0) is a target antigen in some patients with CIDP. ${ }^{61}$ Antibodies to neurofascin and contactin-1, which are concentrated near the nodes of Ranvier, are found in a small population of patients with CIDP.62 In a few patients, IgG4 antibodies to the paranodal proteins contactin and NF155 have been associated with severe, intravenous immunoglobulin (IVIG)-resistant CIDP.63,64 A subset of IVIG-resistant patients with contactin or NF-155 antibodies have responded to rituximab.65,66 This finding supports the premise that improved understanding of antibody responses in CIDP may open new opportunities for future targeted therapeutic interventions.

\section{Conclusions}

CIDP is characterized by progressive weakness and impaired sensory function in the arms and legs, and is caused by demyelination of the peripheral nerves. Different diagnostic criteria are available but without a gold standard. CIDP is a progressive, immune-mediated neuropathy that without treatment can lead to significant disability and in a limited number of patients, death. Comorbid diabetic neuropathy in CIDP patients is an important consideration in clinical deterioration despite adequate immune therapy. IVIG, corticosteroids, and plasmapheresis are first-line therapies for the treatment of CIDP. The choice of a specific therapy for an individual is dictated by several factors, including patient comorbidities and the practice environment. The ICE study indicated benefits for IVIG therapy for reducing disability and functional impairment and improving quality of life. An improved understanding of antibody responses and genetic backgrounds in CIDP may offer new opportunities for targeted interventions.

\section{Important Safety Information}

GAMUNEX-C (immune globulin injection [human], 10\% caprylate/ chromatography purified) is indicated for the treatment of primary humoral immunodeficiency disease (PIDD) in patients 2 years of age and older, idiopathic thrombocytopenic purpura (ITP), and CIDP.

Thrombosis may occur with immune globulin products, including GAMUNEX-C. Risk factors may include: advanced age, prolonged immobilization, hypercoagulable conditions, history of venous or arterial thrombosis, use of estrogens, indwelling central vascular catheters, hyperviscosity, and cardiovascular risk factors. Thrombosis may occur in the absence of known risk factors. For patients at risk of thrombosis, administer GAMUNEX-C at the minimum dose and infusion rate practicable. Ensure adequate hydration in patients before administration. Monitor for signs and symptoms of thrombosis and assess blood viscosity in patients at risk for hyperviscosity.

Renal dysfunction, acute renal failure, osmotic nephrosis, and death may occur with IVIG products in predisposed patients. Patients predisposed to renal dysfunction include those with any degree of preexisting renal insufficiency, DM, age greater than 65, volume depletion, sepsis, paraproteinemia, or patients receiving known nephrotoxic drugs. Renal dysfunction and acute renal failure occur more commonly in patients receiving IVIG products containing sucrose. GAMUNEX-C does not contain sucrose. For patients at risk of renal dysfunction or failure, administer GAMUNEX-C at the minimum concentration available and the minimum infusion rate practicable. 
GAMUNEX-C is contraindicated in patients who have had an anaphylactic or severe systemic reaction to the administration of human immune globulin. It is contraindicated in IgA-deficient patients with antibodies against IgA and history of hypersensitivity.

Severe hypersensitivity reactions may occur with IVIG products, including GAMUNEX-C. In case of hypersensitivity, discontinue GAMUNEX-C infusion immediately and institute appropriate treatment.

Monitor renal function, including blood urea nitrogen (BUN), serum creatinine, and urine output in patients at risk of developing acute renal failure.

Hyperproteinemia, increased serum viscosity, and hyponatremia may occur in patients receiving IVIG treatment, including GAMUNEX-C.

There have been reports of noncardiogenic pulmonary edema (transfusionrelated acute lung injury [TRALI]), hemolytic anemia, and aseptic meningitis in patients administered with IVIG, including GAMUNEX-C.

The high-dose regimen ( $1 \mathrm{~g} / \mathrm{kg} \times 1-2$ days) is not recommended for individuals with expanded fluid volumes or where fluid volume may be a concern.

Because GAMUNEX-C is made from human blood, it may carry a risk of transmitting infectious agents, eg, viruses, the variant Creutzfeldt-Jakob disease (VCJD) agent, and, theoretically, the Creutzfeldt-Jakob disease (CJD) agent.

Do not administer GAMUNEX-C subcutaneously in patients with ITP because of the risk of hematoma formation.

Periodic monitoring of renal function and urine output is particularly important in patients judged to be at increased risk of developing acute renal failure. Assess renal function, including measurement of BUN and serum creatinine, before the initial infusion of GAMUNEX-C and at appropriate intervals thereafter.

Consider baseline assessment of blood viscosity in patients at risk for hyperviscosity, including those with cryoglobulins, fasting chylomicronemia/ markedly high triacylglycerols (triglycerides), or monoclonal gammopathies, because of the potentially increased risk of thrombosis.

If signs and/or symptoms of hemolysis are present after an infusion of GAMUNEX-C, perform appropriate laboratory testing for confirmation.

If TRALI is suspected, perform appropriate tests for the presence of antineutrophil antibodies and anti-HLA antibodies in both the product and patient's serum.

After infusion of IgG, the transitory rise of the various passively transferred antibodies in the patient's blood may yield positive serological testing results, with the potential for misleading interpretation.

In clinical studies, the most common adverse reactions with GAMUNEX-C were headache, fever, chills, hypertension, rash, nausea, and asthenia (in CIDP); headache, cough, injection-site reaction, nausea, pharyngitis, and urticaria with intravenous use (in PIDD) and infusion-site reactions, headache, influenza, fatigue, arthralgia, and pyrexia with subcutaneous use (in PIDD); and headache, vomiting, fever, nausea, back pain, and rash (in ITP).

The most serious adverse reactions in clinical studies were pulmonary embolism (PE) in 1 subject with a history of PE (in CIDP), an exacerbation of autoimmune pure red cell aplasia in 1 subject (in PIDD), and myocarditis in 1 subject that occurred 50 days post-study drug infusion and was not considered drug related (in ITP).

Please see the brief summary of full Prescribing Information for GAMUNEX-C in the Appendix. $\square$
1. National Institute of Neurological Disorders and Stroke (NINDS) Chronic Inflammatory Demyelinating Polyneuropathy (CIDP) Information Page, 2015. Available at: http://www.ninds.nih.gov/ disorders/cidp/cidp.htm (accedded December 3, 2015)

2. Khadilkar SV, Deshmukh SS, Dhonde PD, Chronic dysimmune neuropathies: Beyond chronic demyelinating polyradiculoneuropathy, Annals of Indian Academy of Neurology, 2011:14:81-92.

3. McCombe PA, Pollard JD, McLeod JG, Chronic inflammatory demyelinating polyradiculoneuropathy. A clinical and electrophysiological study of 92 cases, Brain, 1987;110:1617-30

4. Ruts $L$, Drenthen J, Jacobs BC, van Doorn PA, Distinguishing acute-onset CIDP from fluctuating Guillain-Barre syndrome: a prospective study, Neurology, 2010;74:1680-6.

5. American Association of Neuromuscular \& Electrodiagnostic Medicine (AANEM). Chronic Inflammatory Demyelinating Polyneuropathy, 2015. Available at: http://www.aanem.org/ Patients/Disorders/Chronic-Inflammatory-DemyelinatingPolyneuropathy (accessed December 3, 2015)

6. Laughlin RS, Dyck PJ, Melton $\sqcup 3$ rd, et al., Incidence and prevalence of CIDP and the association of diabetes mellitus, Neurology, 2009:73:39-45.

7. Mathey EK, Park SB, Hughes RA, et al., Chronic inflammatory demyelinating polyradiculoneuropathy: from pathology to phenotype, J Neurol Neurosurg Psychiatry, 2015:86:973-85.

8. Dalakas MC, Advances in the diagnosis, pathogenesis and treatment of CIDP, Nat Rev Neurol, 2011;7:507-17.

9. Manso C, Querol L, Mekaouche M, et al., Contactin-1 IgG4 antibodies cause paranode dismantling and conduction defects, Brain, 2016;139:1700-12.

10. Bromberg $\mathrm{MB}$, Comparison of electrodiagnostic criteria for primary demyelination in chronic polyneuropathy, Muscle Nerve, 1991:14:968-76.

11. Brannagan TH 3rd, Current diagnosis of CIDP: the need for biomarkers, J Peripher Nerv Syst, 2011;16 Suppl 1:3-13.
12. Van den Bergh $P Y$, Hadden RD, Bouche $P$, et al., European Federation of Neurological Societies/Peripheral Nerve Society guideline on management of chronic inflammatory demyelinating polyradiculoneuropathy: report of a joint task force of the European Federation of Neurological Societies and the Peripheral Nerve Society - first revision, Eur J Neurol, 2010;17:356-63.

13. Rajabally YA electrophysiology in the diagnosis of chronic inflammatory demyelinating polyneuropathy: a study of 20 cases, $J$ Peripher Nerv Syst, 2005;10:282-92.

14. Vo ML, Hanineva A, Chin RL, et al., Comparison of 2-limb versus 3-limb electrodiagnostic studies in the evaluation of chronic inflammatory demyelinating polyneuropathy, Muscle Nerve, 2015;51:549-53.

15. Rajabally YA Nicolas $G$, Pieret $F$ et al, validity of diagnostic criteria for chronic inflammatory demyelinating polyneuropathy: a multicentre European study, J Neurol Neurosurg Psychiatry, 2009:80:1364-8.

16. Koski $C L$, Baumgarten $M$, Magder $L S$, et al., Derivation and validation of diagnostic criteria for chronic inflammatory demyelinating polyneuropathy, J Neurol Sci, 2009:277:1-8.

17. Breiner $\mathrm{A}$, Brannagan $\mathrm{TH}$ 3rd, Comparison of sensitivity and specificity among 15 criteria for chronic inflammatory demyelinating polyneuropathy Muscle Nerve, 2014:50:40-6.

18. Chio A, Plano F, Calvo A, et al., Comorbidity between CIDP and diabetes mellitus: only a matter of chance?, Eur J Neurol, 2009;16:752-4

19. Sharma KR, Cross J, Farronay O, et al., Demyelinating neuropathy in diabetes mellitus, Arch Neurol, 2002;59:758-65.

20. Russell JW, Zilliox LA, Diabetic neuropathies, Continuum (Minneapolis, Minn), 2014;20(5 Peripheral Nervous System Disorders):1226-40.

21. Garces-Sanchez M, Laughlin RS, Dyck PJ, et al., Painless diabetic motor neuropathy: a variant of diabetic lumbosacra radiculoplexus Neuropathy?, Ann Neurol, 2011;69:1043-54.
22. Dunnigan SK, Ebadi H, Breiner A, et al., The characteristics of chronic inflammatory demyelinating polyneuropathy in patients with and without diabetes-an observational study, Plos One, 2014;9:e89344

23. Dimachkie MM, Barohn RJ, Chronic inflammatory demyelinating polyneuropathy, Curr Treat Options Neurol, 2013;15:350-66.

24. Allen JA, Lewis RA, CIDP diagnostic pitfalls and perception of treatment benefit, Neurology, 2015;85:498-504.

25. Kaplan A, Brannagan TH 3rd, Evaluation of Patients with Refractory Chronic Inflammatory Demyelinating Polyneuropathy, Muscle Nerve, 2016; Epub ahead of print.

26. Magda P, Latov N, Brannagan TH 3rd, et al., Comparison of electrodiagnostic abnormalities and criteria in a cohort of patients with chronic inflammatory demyelinating polyneuropathy, Arch Neurol, 2003;60:1755-9.

27. Hughes RA, Cornblath DR. Guillain-Barre syndrome, Lancet, 2005:366:1653-66.

28. Eldar AH, Chapman J, Guillain Barre syndrome and other immune mediated neuropathies: diagnosis and classification, Autoimmun Rev, 2014;13:525-30.

29. Kerasnoudis A, Pitarokoili K, Haghikia A, et al., Nerve ultrasound protocol in differentiating chronic immune-mediated neuropathies, Muscle Nenve 2016:54:864-71

30. Katz JS, Saperstein DS, Gronseth G, et al., Distal acquired demyelinating symmetric neuropathy, Neurology, 2000;54:615-20

31. Willison $\mathrm{HJ}, \mathrm{O}^{\prime} \mathrm{L}$ eary $\mathrm{CP}$, Veitch J, et al., The clinical and laboratory features of chronic sensory ataxic neuropathy with anti-disialosy IgM antibodies, Brain, 2001;124:1968-77.

32. Nasu S, Misawa S, Sekiguchi Y, et al., Different neurological and physiological profiles in POEMS syndrome and chronic inflammatory demyelinating polyneuropathy, J Neurol Neurosurg inflammatory demyelinating

33. European Federation of Neurological Societies/Peripheral Nerve Society Guideline on management of paraproteinemic 
demyelinating neuropathies. Report of a Joint Task Force of the European Federation of Neurological Societies and the Peripheral Nerve Society-first revision, J Peripher Nerv Syst, 2010;15:185-95.

34. Dispenzieri A, POEMS syndrome: 2014 update on diagnosis, riskstratification, and management, Am J Hematol, 2014:89:214-23.

35. Soubrier MJ, Dubost JJ, Sauvezie BJ, POEMS syndrome: a study of 25 cases and a review of the literature. French Study Group on POEMS Syndrome, Am J Med, 1994; $97: 543-53$

36. Mauermann ML, Sorenson EJ, Dispenzieri A, et al., Uniform demyelination and more severe axonal loss distinguish POEMS syndrome from CIDP, I Neurol Neurosurg Psychiatry, 2012;83:480-6.

37. Watanabe $\mathrm{O}$, Maruyama I, Arimura K, et al., Overproduction of vascular endothelial growth factor/vascular permeability factor is causative in Crow-Fukase (POEMS) syndrome, Muscle Nerve, 1998;21:1390-7.

38. Briani C, Fabrizi GM, Ruggero S, et al., Vascular endothelial growth factor helps differentiate neuropathies in rare plasma cell dyscrasias, Muscle Nerve, 2011;43:164-7.

39. Nobile-Orazio E, Terenghi F, Giannotta $\mathrm{C}$, et al., Serum VEGF levels in POEMS syndrome and in immune-mediated neuropathies, Neurology, 2009;72:1024-6.

40. Dyck PJ, O'Brien PC, Oviatt KF, et al., Prednisone improves chronic inflammatory demyelinating polyradiculoneuropathy more than no treatment, Ann Neurol, 1982;11:136-41.

41. Hughes RA, Mehndiratta MM, Corticosteroids for chronic inflammatory demyelinating polyradiculoneuropathy, cochrane Database Syst Rev, 2015;1:Cd002062.

42. van Schaik IN, Eftimov F, van Doorn PA, et al., Pulsed high-dose dexamethasone versus standard prednisolone treatment for chronic inflammatory demyelinating polyradiculoneuropathy (PREDICT study): a double-blind, randomised, controlled trial, Lancet Neurol, 2010;9:245-53.

43. Sabatelli M, Madia F, Mignogna T, et al., Pure motor chronic inflammatory demyelinating polyneuropathy, J Neurol, 2001;248:772-7.

44. Molenaar DS, van Doorn PA, Vermeulen M, Pulsed high dose dexamethasone treatment in chronic inflammatory demyelinating polyneuropathy: a pilot study, I Neurol Neurosurg Psychiatry,
1997;62:388-90.

45. Gorson KC, An update on the management of chronic inflammatory demyelinating polyneuropathy, Ther Adv Neurol Disord, 2012:5:359-73.

46. Hughes RA, Donofrio P, Bril V et al., Intravenous immune globulin (10\% caprylate-chromatography purified) for the treatment of chronic inflammatory demyelinating polyradiculoneuropathy (ICE study): a randomised placebo-controlled trial, Lancet Neurol, 2008;7:136-44

47. Merkies IS, Schmitz PI, van der Meche FG, van Doorn PA, Psychometric evaluation of a new sensory scale in immunemediated polyneuropathies. Inflammatory Neuropathy Cause and Treatment (INCAT) Group, Neurology, 2000:54:943-9.

48. Vanhoutte EK, Latov N, Deng C, et al. Vigorimeter grip strength in CIDP: a responsive tool that rapidly measures the effect of IVIGthe ICE study, Eur I Neurol, 2013;20:748-55.

49. Deng C, Hanna K, Bril V, et al., Challenges of clinical trial design when there is lack of clinical equipoise: use of a responseconditional crossover design, I Neurol, 2012:259:348-52.

50. Merkies IS, van Nes SI, et al., Confirming the efficacy of intravenous immunoglobulin in CIDP through minimum clinically important differences: shifting from statistical significance to clinical relevance, I Neurol Neurosurg Psychiatry, 2010;81:1194-9.

51. Orbach H, Katz U, Sherer $Y$, Shoenfeld $Y$, Intravenous immunoglobulin: adverse effects and safe administration, Clin Rev Allergy Immunol, 2005;29:173-84.

52. Merkies IS, Bril V, Dalakas MC, et al., Health-related quality-of-life improvements in CIDP with immune globulin IV 10\%: the ICE Study, Neurology, 2009:72:1337-44.

53. GAMUNEX ${ }^{\circledR}-\mathrm{C}$ (immune globulin injection [human], 10\% caprylate/ chromatography purified) Prescribing Information. Grifols Available at: www.fda.gov.tw/MLMS/ShowFile.aspx? Licld $=10000796 \&$ Seq $=013 \&$ Type $=9$ (accessed October 26, 2016)

54. Dyck PJ, Daube J, O'Brien P, et al., Plasma exchange in chronic inflammatory demyelinating polyradiculoneuropathy N Eng/ 」 Med, 1986;314:461-5.

55. Hahn AF, Bolton CF, Pillay N, et al., Plasma-exchange therapy in chronic inflammatory demyelinating polyneuropathy.
A double-blind, sham-controlled, cross-over study, Brain, 1996;119(Pt 4):1055-66.

56. Mehndiratta MM, Hughes RA, Pritchard J, Plasma exchange for chronic inflammatory demyelinating polyradiculoneuropathy, Cochrane Database Syst Rev, 2015:Cd003906.

57. Good JL, Chehrenama M, Mayer RF, Koski CL, Pulse cyclophosphamide therapy in chronic inflammatory demyelinating polyneuropathy, Neurology, 1998; 51:1735-8.

58. Dyck PJ, O'Brien P, Swanson C, et al., Combined azathioprine and prednisone in chronic inflammatory-demyelinating polyneuropathy, Neurology, 1985;35:1173-6.

59. Mahdi-Rogers M, Swan AV, van Doorn PA, Hughes RA, Immunomodulatory treatment other than corticosteroids, immunoglobulin and plasma exchange for chronic inflammatory demyelinating polyradiculoneuropathy, Cochrane Database Syst Rev, 2010:Cd003280

60. Chin RL, Deng C, Bril V, et al., Follow-up nerve conduction studies in CIDP after treatment with IGIV-C: Comparison of patients with and without subsequent relapse, Muscle Nerve, 2015:52:498-502.

61. Allen D, Giannopoulos K, Gray I, et al., Antibodies to peripheral nerve myelin proteins in chronic inflammatory demyelinating polyradiculoneuropathy, J Peripher Nerv Syst, 2005;10:174-80.

62. Kawamura N, Yamasaki R, Yonekawa T, et al., Anti-neurofascin antibody in patients with combined central and periphera demyelination, Neurology, 2013;81:714-22

63. Ng JK, Malotka J, Kawakami N, et al., Neurofascin as a target for autoantibodies in peripheral neuropathies, Neurology, 2012;79:2241-8.

64. Querol L, Nogales-Gadea G, Rojas-Garcia R, et al., Neurofascin IgG4 antibodies in CIDP associate with disabling tremor and poor response to IVIg, Neurology, 2014;82:879-86.

65. Querol L, Nogales-Gadea G, Rojas-Garcia R, et al., Antibodies to contactin-1 in chronic inflammatory demyelinating polyneuropathy, Ann Neurol, 2013;73:370-80.

66. Querol L, Rojas-Garcia R, Diaz-Manera J, et al., Rituximab in treatment-resistant CIDP with antibodies against paranodal proteins, Neurol Neuroimmunol Neuroinflamm, 2015:2:149. 